\title{
A Spectrophotometric Technique for Recording Uptake of an Organomercurial by Mycelial Fungi
}

\author{
By W. GREENAWAY, S. WARD, A. K. RAJAN AND F. R. WHATLEY \\ Botany School, South Parks Road, Oxford OX1 3RA
}

(Received 11 January 1978)

\begin{abstract}
A spectrophotometric technique is described by which uptake of 2-chloromercuri-4-nitrophenol (CMNP), an organomercurial compound toxic to fungi, may be continuously monitored for 30 min periods. The method is demonstrated using mercury-resistant and mercury-susceptible isolates of Penicillium expansum and Cladosporium cladosporioides. With $P$. expansum no consistent differences in uptake were recorded. Mercury-resistant isolates of $C$. cladosporioides consistently absorbed less CMNP into their hyphae than did mercury-susceptible isolates.
\end{abstract}

\section{INTRODUCTION}

Development of resistance to organomercurial fungicides in vivo has been demonstrated in a number of fungal genera including Aspergillus (Gibson, 1953), Chrysosporium (Williams \& Pugh, 1975), Leptosphaeria (Harrower, 1976), Pyrenophora (Noble et al., 1966), Cladosporium, Syncephalastrum (Greenaway, 1972a), Ulocladium (Greenaway, 1973b) and Penicillium (Kiessling, 1961; Greenaway, Cripps \& Ward, 1974).

Resistance to these compounds may be due to exclusion of the mercurial ions from the hyphae, to active excretion of mercurial ions which have entered the hyphae, or to detoxification of the compound within the hyphae. We therefore wished to measure the uptake of mercurials by mercury-resistant and mercury-susceptible fungi to see whether differential uptake occurred and have examined a colorimetric technique by which such uptake might be monitored.

Measurement of the decrease in absorbance $(A)$ of a compound can provide a convenient method of monitoring uptake from aqueous solutions. This method is unfortunately difficult to apply to most organomercurial compounds which are used as fungicides, e.g. phenyl$\mathrm{Hg}-\mathrm{X}$, ethyl-Hg-X, because these compounds absorb light maximally at low wavelengths $(<340 \mathrm{~nm})$ and fungi often release into the solution materials which also absorb at these wavelengths. However, the organomercurial 2-chloromercuri-4-nitrophenol (CMNP), which is not a commercial fungicide, has an absorption peak at $405 \mathrm{~nm}$. This compound was found to be toxic to fungi in vitro and was used for studies comparing uptake by resistant and susceptible isolates of $C$. cladosporioides and $P$. expansum.

\section{METHODS}

Isolates, media and growth. The fungal isolates used were: Penicillium expansum Link ex S. F. Gray emend. Thom, mercury-susceptible s139 and mercury-resistant R143; Cladosporium cladosporioides (Fres.) de Vries, mercury-susceptible s37, s38, s39 and mercury-resistant $\mathrm{R} 251, \mathrm{R} 252$, $\mathrm{R} 253$. The isolates were maintained on minimal-malt agar (Greenaway, 1973a) at $20{ }^{\circ} \mathrm{C}$ and spores obtained from colonies grown under these conditions were used for inoculation of liquid media (minimal-malt without agar but containing 25 p.p.m. streptomycin sulphate). 


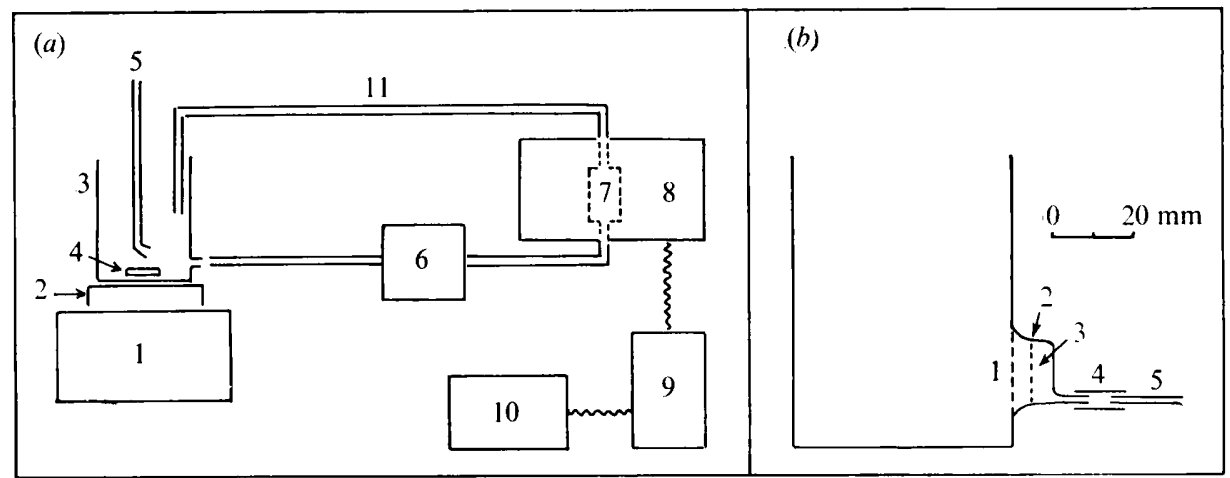

Fig. 1. (a) Instrumentation for recording uptake: 1, magnetic stirrer; 2, plastic Petri-dish lid; 3 , reservoir; 4, magnetic stirring bar; 5, aerator; 6, peri-pump; 7, flow-through cell; 8, SP600 UV spectrophotometer; 9 , linear-log converter; 10 , recorder; 11 , Teflon tubing.

(b) Reservoir for uptake experiments: 1, perforated glass plate; 2 , sinter disc; 3 , air trap; 4, silicone rubber tubing connector; 5 , Teflon tubing.

Media were autoclaved at $121{ }^{\circ} \mathrm{C}$ for $15 \mathrm{~min}$. In tests for resistance, CMNP in $0 \cdot 1 \mathrm{M}-\mathrm{KOH}$ was added to autoclaved minimal-malt medium cooled to $50^{\circ} \mathrm{C}$.

Mycelium for uptake experiments was grown in $500 \mathrm{ml}$ of minimal-malt liquid medium in 21 conical flasks. Medium was inoculated with a spore suspension, sufficient to produce about 100 mycelial pellets per flask, and incubated in a Gallenkamp cooled orbital incubator at $20^{\circ} \mathrm{C}$ and $160 \mathrm{rev}$. $\mathrm{min}^{-1}$ for $6 \mathrm{~d}(C$. cladosporioides) or $5 \mathrm{~d}(P$. expansum). This produced mycelial pellets of about $5 \mathrm{~mm}$ diam. Some isolates (e.g. $C$. cladosporioides $\mathrm{R} 251$ and $\mathrm{R} 253$ ) grew more rapidly in liquid medium than others of the same species and in these cases fewer mycelial pellets were used in uptake experiments because they were bigger.

During uptake experiments, mycelial pellets were maintained in a solution containing $0 \cdot 1 \mathrm{M}$-glucose (to provide a respiratory substrate for the fungus) and $\mathrm{Na}_{2} \mathrm{HPO}_{4} / \mathrm{NaH}_{2} \mathrm{PO}_{4}(40 \mathrm{~mm}, \mathrm{pH} 6 \cdot 8)$ which was essential to stabilize the pH, since absorbance of CMNP at $405 \mathrm{~nm}$ is $\mathrm{pH}$ dependent.

Apparatus for spectrophotometric assessment of uptake. The mycelial pellets suspended in buffered glucose solution containing CMNP were incubated in a reservoir. The uptake was continuously monitored by circulating the suspending liquid from the reservoir through a Hellma semi-micro flow-through cell (no. 134) mounted in a Pye Unicam SP600 UV spectrophotometer (set at $405 \mathrm{~nm}$ ) and back to the reservoir through narrow Teflon tubing (Fig. $1 a$ ). Small sections of silicone rubber tubing were used for joints. The solution was circulated by a peri-pump (Orme Scientific) fitted with a $60 \mathrm{rev}$. min $^{-1}$ motor and a $2.5 \mathrm{~mm}$ silicone rubber tube giving a flow of $12 \mathrm{ml} \mathrm{min}{ }^{-1}$. The total volume of liquid was $50 \mathrm{ml}$ of which $3 \cdot 0 \mathrm{ml}$ was outside the reservoir at any one time.

The reservoir was fitted with a $15 \mathrm{~mm}$ diam. sinter disc, porosity 0 , mounted behind a glass plate $(15 \mathrm{~mm}$ diam.) perforated with numerous $1 \mathrm{~mm}$ holes. The perforated plate prevented the mycelium from contacting the sinter disc, which otherwise became rapidly blocked. The outlet from the reservoir had a small trap above it (Fig. $1 b$ ) to collect air bubbles which might otherwise have accumulated in the spectrophotometer cell. Air was bubbled into the buffered glucose solution through a sinter disc at $20 \mathrm{ml} \mathrm{min} \mathrm{m}^{-1}$ and the mycelial pellets were kept in motion by a slowly revolving Teflon-coated magnetic stirring bar. The reservoir stood on a plastic Petri-dish lid to insulate it from the slight heating effect of the stirrer.

The logarithmic signal of absorption produced by the spectrophotometer was converted by a Pye Unicam SP45 linear-log converter to a linear signal, which was recorded continuously on a Smiths Servoscribe potentiometric recorder.

Experimental procedure for assessment of uptake. Buffered glucose solution was circulated through the system to check that it was functioning efficiently. The solution was then replaced by $50 \mathrm{ml}$ of buffered glucose solution containing $60 \mu \mathrm{M}-\mathrm{CMNP}$ and this solution was circulated for $5 \mathrm{~min}$ to ensure a stable recorder baseline. No adsorption of CMNP by the circulation system was recorded during a 30 min test period.

Mycelial pellets were strained from the growth medium, rinsed in buffered glucose solution for $30 \mathrm{~min}$ in the orbital incubator and drained in a nylon bag. A $10 \mathrm{ml}$ volume (about 50 to 80 mycelial pellets) was added to the mercurial solution and stirred gently for $30 \mathrm{~min}$, and the $A_{405}$ was continuously recorded. The $\mathrm{pH}$ of the mercurial solution did not change during this period.

At the end of the uptake period we investigated the distribution of CMNP that was removed from the circulating solution by the mycelial pellets. The mycelial pellets were removed from the solution, rinsed for 5 min with gentle stirring and aeration in $30 \mathrm{ml}$ of buffered glucose solution (to remove any CMNP loosely 

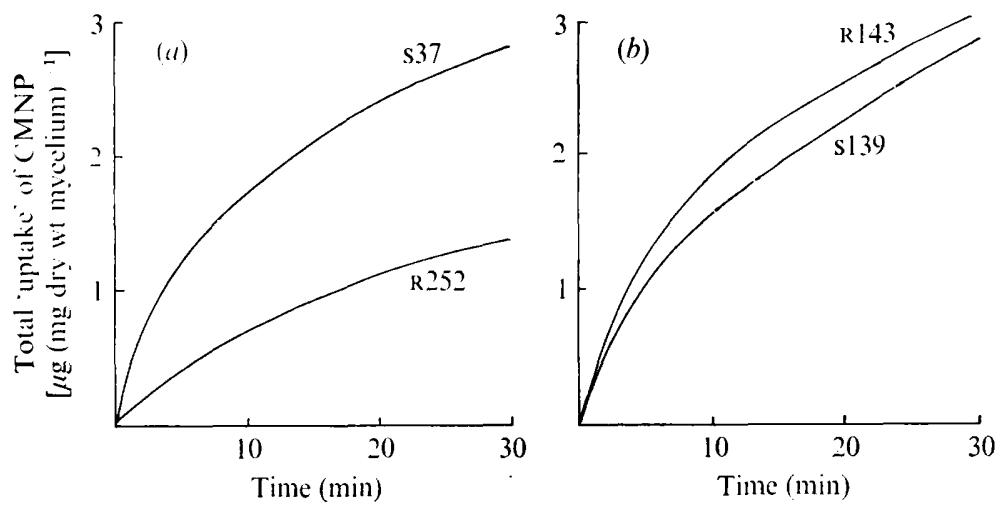

Fig. 2. Removal of 2-chloromercuri-4-nitrophenol from solution by mercury-resistant and mercurysusceptible isolates. (a) Cladosporium cladosporioides $\mathbf{s} 37$ (susceptible), dry wt of mycelium $130 \mathrm{mg}$; R252 (resistant), dry wt of mycelium $122 \mathrm{mg}$. (b) Penicillium expansum s139 (susceptible), dry wt of nivcelium $69 \cdot 2 \mathrm{mg} ; \mathrm{R} 143$ (resistant), dry wt of mycelium $102 \mathrm{mg}$.

associated with the hyphae) and then for $5 \mathrm{~min}$ in $30 \mathrm{ml}$ of $5 \mathrm{~mm}$-cysteine solution, freshly made and adjusted to $\mathrm{pH} 8.0$ with $0.5 \mathrm{M}-\mathrm{KOH}$ (to remove CMNP more tightly bound to the hyphae).

The amounts of CMNP removed from the mycelial pellets by rinse solutions were assessed by measurement of $A_{405}$ after adjusting the solutions to $0.1 \mathrm{M}$ with respect to $\mathrm{KOH}$, because the $A_{405}$ of CMNP is at its highest in $0.1 \mathrm{M}-\mathrm{KOH}$ (McMurray \& Trentham, 1969), and also because the CMNP-cysteine complex is apparently destroyed in $0 \cdot 1 \mathrm{M}-\mathrm{KOH}$, thus making the two measurements directly comparable.

Measurement of interhyphal spaces in the mycelial pellet. The volume of the spaces between the hyphae of a mycelial pellet may affect uptake and rinsing patterns. The interhyphal spaces were therefore measured using blue dextran (Greenaway \& Ward, 1978).

\section{RESULTS}

\section{Effect of 2-chloromercuri-4-nitrophenol on mycelial growth}

The resistant $P$. expansum isolate grew at $65 \%$ of control growth in the presence of the CMNP at $80 \mu \mathrm{M}$ and at $35 \%$ of control growth at $160 \mu \mathrm{M}$. The susceptible isolate did not grow at concentrations above $80 \mu \mathrm{M}$. The resistant $C$. cladosporioides isolate $\mathrm{R} 252$ grew at $45 \%$ of the control growth in the presence of CMNP at $80 \mu \mathrm{M}$ and at $10 \%$ of the control growth at $160 \mu \mathrm{M}$. The other resistant isolates (R251 and R253) behaved similarly. The susceptible $C$. cladosporioides isolates (s37, s38 and s39) grew hardly at all at $80 \mu \mathrm{M}$.

\section{Uptake of 2-chloromercuri-4-nitrophenol}

Susceptible isolates of $C$. cladosporioides took up CMNP more rapidly than did resistant isolates, particularly during the first $5 \mathrm{~min}$ of uptake. Figure 2(a) shows results for susceptible (s37) and resistant ( $\mathrm{R} 252)$ isolates. On the basis of a set of six replicates, the total 'uptake' (in $30 \mathrm{~min}$ ) by C. cladosporioides $\mathrm{R} 252$ was $1.78 \pm 0.30 \mu \mathrm{g}$ (mg dry wt mycelium) $)^{-1}$ and the uptake by s37 was $2.88 \pm 0.29 \mu \mathrm{g}$ (mg dry wt mycelium) ${ }^{-1}$; these values are significantly different at the $5 \%$ level. For other isolates of $C$. cladosporioides, the average uptake (three replicates of each) was 1.54 and $0.95 \mu \mathrm{g}$ (mg dry wt mycelium) ${ }^{-1}$ for R251 and $\mathrm{R} 253$, respectively, and 2.09 and $2.41 \mu \mathrm{g}$ (mg dry wt mycelium) ${ }^{-1}$ for s38 and s39, respectively. Thus, on average, the susceptible isolates of $C$. cladosporioides took up $74 \%$ more CMNP than did resistant isolates. Uptake of CMNP by the resistant and susceptible isolates of $P$. expansum is shown in Fig. $2(b)$. On the basis of a set of 10 replicates, the uptake of CMNP by the resistant isolate of $P$. expansum, R143, was $3.56 \pm 0.84 \mu \mathrm{g}$ (mg dry wt mycelium) $)^{-1}$ and the uptake by the susceptible isolate, $\mathrm{s} 139$, was $2.67 \pm 0.67 \mu \mathrm{g}$ (mg dry wt mycelium) ${ }^{-1}$; these values are not significantly different.

The total 'uptake' included_CMNP that had passed into the space between the hyphae 
(a dilution effect which perhaps shows up as the initial rapid 'uptake' in Fig. 2), that which became associated with wall constituents and that which was taken up into the cytoplasm. By rinsing the mycelial pellets that had taken up CMNP, first with buffered glucose and then with cysteine solutions, the distribution of the CMNP within the different compartments could be assessed. The amounts of CMNP released from both the resistant and susceptible isolates of $P$. expansum by glucose and cysteine rinses were similar, and similar amounts remained after the rinses. With the $C$. cladosporioides isolates, however, a larger percentage of the CMNP associated with the mycelial pellets was removed by the glucose rinse from the resistant isolates $(\mathrm{R} 251,11 \% ; \mathrm{R} 252,15 \% ; \mathrm{R} 253,18 \%)$ than from the susceptible isolates $(\mathrm{s} 37,11 \% ; \mathrm{s} 38,2 \% ; \mathrm{s} 39,4 \%)$. Cysteine rinses removed similar percentages of the remaining CMNP from all isolates. After the rinses, the CMNP associated with resistant $C$. cladosporioides isolates was on average less than half that associated with susceptible isolates.

The blue dextran-permeable space of mycelial pellets of resistant isolates of $C$. cladosporioides was smaller (approx. $1.9 \mathrm{ml}$ in $10 \mathrm{ml}$ pellet volume) than that in susceptible isolates (approx. $4 \cdot 1 \mathrm{ml}$ in $10 \mathrm{ml}$ pellet volume). The blue dextran-permeable space could not be accurately measured in P. expansum (see Greenaway \& Ward, 1978) but was close to $5 \mathrm{ml}$ in $10 \mathrm{ml}$ pellet volume. There appeared to be no obvious difference between resistant and susceptible isolates in this respect.

\section{DISCUSSION}

The apparatus described here for circulation of CMNP solution through a spectrophotometer is similar to that described by Haverkate, Brevoord \& Verloop (1972). Our reservoir is of a simpler construction than that of Haverkate and co-workers, but our filter system would need modification for uptake studies using fungal spores or bacteria. Continuous recording of uptake for $30 \mathrm{~min}$ was achieved with ease and provided a direct recording of the decrease in concentration of CMNP in the solution to which mycelial pellets had been added.

The method we describe has the advantage that it permits any changes in the kinetics of uptake during the experimental period to be clearly seen. From Fig. 2 we may speculate that the initial more rapid 'uptake' represents the initial equilibration between the suspending solution and the measured blue dextran-permeable space. The extent of the initial uptake appears to be related to this space.

The $A_{405}$ of a solution of CMNP containing mycelium could also be monitored by periodically removing samples of the liquid, filtering through a sinter to remove detached hyphae and reading the $A_{405}$, samples then being returned to the solution containing mycelium. Such a procedure yields similar results to those presented here, but does not give a continuous record. A previous technique using dithizone to assess uptake of phenyl-mercury by Pyrenophora avenae Ito \& Kuribay. (Greenaway, 1972b) is much less convenient than that described here.

As there was no consistent difference in amounts of CMNP associated with mycelium of resistant and susceptible $P$. expansum isolates, either before or after rinsing, resistance is presumed to depend either on metabolic detoxification or on the binding of mercurials to inessential metabolites within the hyphae which may prevent their access to the site of action within the cell. In contrast, less CMNP was usually associated with mycelial pellets of resistant isolates of $C$. cladosporioides than with those of susceptible isolates. The difference in uptake by these isolates appears to be related to differences in the measured blue dextranpermeable volume, both being smaller in resistant than in susceptible isolates. Although our experiments do not of course relate to natural conditions, they do suggest that a difference in the growth habit may result in the uptake of less organomercurial fungicide by resistant isolates of $C$. cladosporioides.

We have examined the walls of both resistant and susceptible $C$. cladosporioides using gas-liquid chromatography and infrared techniques but have detected no differences in 
their structure. Minor changes in structure would, however, not have been detected by these techniques.

This work was supported by a grant from the Agricultural Research Council.

\section{REFERENCES}

Gibson, I. A. S. (1953). Crown rot, a seedling disease of ground nuts caused by Aspergillus niger. 2. An anomalous effect of organo-mercury seed dressings. Transactions of the British Mycological Society 36, 324-334.

GreENAWAY, W. (1972a). Epiflora of oat seed treated with organo-mercury. Transactions of the British Mycological Society 58, 321-327.

Greenaway, W. (1972b). Permeability of phenyl$\mathrm{Hg}^{+}$-resistant and phenyl- $\mathrm{Hg}^{+}$-susceptible isolates of Pyrenophora avenae to the phenyl- $\mathrm{Hg}^{+}$ion. Journal of General Microbiology 73, 251-255.

Greenaway, W. (1973a). In vitro tests of the toxicity of mercury compounds to Pyrenophora avenae. Transactions of the British Mycological Society 60, 87-97.

GREENAWAY, W. (1973b). Resistance of Ulocladium atrum to phenyl-mercury. Transactions of the British Mycological Society 60, 359-360.

Greenaway, W. \& Ward, S. (1978). Use of blue dextran to measure interhyphal spaces in pellets of fungal mycelium. Transactions of the British Mycological Society 70, 309-312.

Greenaway, W., Cripps, A. \& Ward, S. (1974). Resistance to organo-mercury by penicillia isolated from cereal seed. Transactions of the British Mycological Society 63, 137-141.
Harrower, K. M. (1976). Tolerance of Leptosphaeria nodorum to an organomercurial compound. Transactions of the British Mycological Society 66, 523-525.

Haverkate, F., Brevoord, J. W. \& Verloop, A. (1972). Automatic recording of uptake of pesticides and related compounds by small plant parasites. 1. Description of the method. Pesticide Science 3, 1-11.

KIESSLING, H. (1961). Some factors concerning the detoxification of organic mercurial fungicides. Svensk papperstidning 64, 689-693.

McMurray, C. H. \& Trentham, D. R. (1969). A new class of chromophoric organomercurials and their reactions with D-glyceraldehyde-3-phosphate dehydrogenase. Biochemical Journal 115, 913-921.

Noble, M., Macgarvie, Q.D., Hams, A. F. \& LEAFE, E. L. (1966). Resistance to mercury of Pyrenophora avenae in Scottish seed oats. Plant Pathology 15, 23-28.

Williams, J. I. \& PUGH, G. J. F. (1975). Resistance of Chrysosporium pannorum to an organomercury fungicide. Transactions of the British Mycological Society 64, 255-263. 